\title{
Operating room efficiency and timing during coronavirus disease 2019 outbreak in a referral orthopaedic hospital in Northern Italy
}

\author{
Mauro Andreata ${ }^{1}$ (D) Martina Faraldi ${ }^{2} \cdot$ Eugenia Bucci $^{3} \cdot$ Giovanni Lombardi $^{2,4} \cdot$ Luigi Zagra $^{1}$ \\ Received: 13 May 2020 / Accepted: 4 August 2020 / Published online: 16 August 2020 \\ (C) SICOT aisbl 2020
}

\begin{abstract}
Purpose The SARS-CoV-2 outbreak affected health care systems at different levels with important consequences on health, economy, and social structures. This paper aims to analyse the impact on surgical block utilisation and efficiency in an orthopaedics reference centre in Northern Italy.

Methods The timeframe chosen for the current analysis was April 2020, to be compared with the corresponding period in 2019. Number and type of procedures, first case delay, occupancy rate, and turnover time were used as indicators to benchmark the activities.

Results The overall number of surgical procedures decreased by 57\%, from 537 in 2019 to 230 in the same timeframe in 2020. Orthopaedic procedures predominated in 2019, with 434 cases $(80.8 \%$ ), while in 2020, trauma was the leading activity, with 200 cases $(86.9 \%)$. Orthopaedic surgery had a relative decrement of $93 \%$ while trauma has relatively increased by $94 \%$. Mean first case delay in orthopaedic room (OR) was two hours and 36 minutes (SD 01:40:21) in 2020 compared with only 19 minutes (SD 00:02:15) in 2019. OR occupancy rate was 59\% in 2020 compared with $89 \%$ in 2019 . Turnover time raised from 21 minutes in 2019 to $53 \mathrm{~min}$ in 2020 .

Conclusions These data clearly show the deep impact of COVID-19 pandemic on OR facilities. Efficiency indicators fell dramatically in April 2020 compared with the corresponding period in 2019. This scenario will deeply affect both the waiting lists and the economic burden of the hospital. Regaining efficiency and maintaining the quality and safety of the process while restoring elective orthopaedic surgery are among the main challenges that surgeons will face in the next time.
\end{abstract}

Keywords COVID-19 $\cdot$ Coronavirus · Orthopaedic trauma $\cdot$ Operating room $\cdot$ Performance indicators $\cdot$ Efficiency

\section{Introduction}

SARS-CoV-2 outbreak had in March and April 2020 the main epicentre in Europe. The first and the worst affected area was Lombardia, a highly populated and industrialised area of Northern Italy, inhabited by 10 million people with a well-

Luigi Zagra

luigi.zagra@fastwebnet.it

1 IRCCS Istituto Ortopedico Galeazzi Hip Department, Milan, Italy

2 Laboratory of Experimental Biochemistry \& Molecular Biology, IRCCS Istituto Ortopedico Galeazzi, Milan, Italy

3 IRCCS Istituto Ortopedico Galeazzi Chief Operating Officer Staff, Milan, Italy

4 Department of Athletics, Strength and Conditioning, Poznań University of Physical Education, Poznań, Poland structured hospital health system. There are over 200 among public and private accredited hospitals and 18 research hospitals (IRCCS). About $22 \%$ of beds are in private hospitals, either profit or not-for-profit.

As of 30 April 2020, the total number of patients affected by COVID-19 in the region was 75,732 (37\% of the overall national burden) with a death toll accounting for 13,772 victims (49\% of the overall national burden) $[1,2]$. The regional hospital network had to change abruptly to comply with the high demand for hospitalisation and the sudden need for intensive care units. On March 2020, the regional government decided to restructure the hospital services by dramatically reducing orthopaedic room (OR) activities and increasing the availability of sub-intensive and intensive care units (ICU). A hub-spoke system was built up to centralise urgent cases needing specialised care as for cardiology, oncology, and even orthopaedic conditions, while SARS-CoV-2 spreading was reaching the climax. 
Regarding orthopaedic and trauma services, a hub scheme was established. Poly-trauma centres were reduced from six to three. In Milano, two referral orthopaedic centres were identified as hubs for uncomplicated trauma and non-deferrable orthopaedic procedures, i.e. oncological and infectious orthopaedic conditions [3].

Galeazzi Orthopaedic Institute is an orthopaedic research centre performing the highest number of surgical orthopaedic procedures in Italy. The normal activity includes 21 units (including surgery, rehabilitation, and complementary clinics) for a total of 364 beds; two surgery blocks for major surgery and one surgery block for day-surgery. The yearly activity accounts for an average of 14,600 including more than 4000 joint arthroplasties, 1400 major spine procedures, and 1200 trauma procedures.

During the pandemic, the entire elective surgery activity has been stopped. Galeazzi Orthopaedic Institute was chosen by the Regional Health Authorities as a hub hospital for both orthopaedics and minor trauma surgery [4]. Patients from surrounding hospitals were redirected to our centre. Moreover, at the peak of the outbreak, the hospital treated also nonorthopaedic SARS-CoV-2-positive patients to contribute to the general need of COVID wards [5].

Based on these urgent needs, Galeazzi Orthopaedic Institute was reorganised by defining distinct routes for
SARS-CoV-2-positive and SARS-CoV-2-negative patients (Fig. 1)

One of the two major surgery blocks was converted into an intensive care unit (ICU) while the other block was divided into two distinct areas to keep physically separated the procedures for SARS-CoV-2-positive (OR .6.1 and 6.2) and SARSCoV-2-negative patients (OR 6.5, 6.6, 6.7). In this scenario, part of the theatre nurses was redeployed in the new ICU while two nurses for each active OR reinforced the staff to assist in doffing and donning and sustaining scrub nurses. Since the total number of ORs decreased, we did not suffer staff shortage

This study aimed to analyse the impact of COVID-19 pandemic on surgical room utilisation and efficiency within an operating block in a reference orthopaedic centre during April 2020.

\section{Materials and methods}

The internal administrative flows integrated and doublechecked by the health management data of the hospital represented the data sources of this study. Approval was obtained by the institutional review board for using anonymous data flows in an aggregated form.

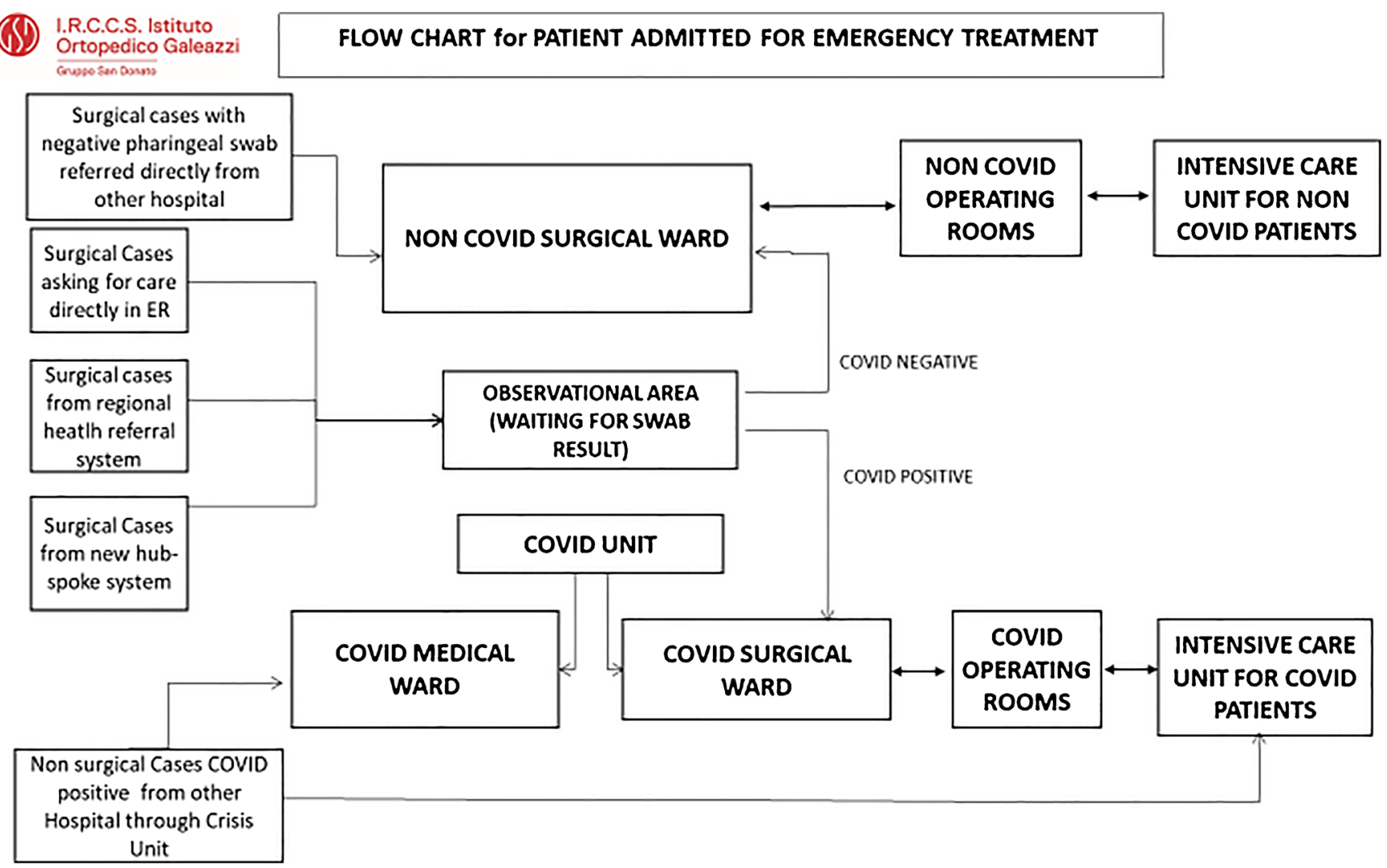

Fig. 1 Flow chart of admissions in emergency room 
The timeframe chosen for the analysis was represented by the first four weeks of April (1 to 28 April 2020), to be compared with the corresponding period in 2019.

During this period, daily surgical activities were planned according to the non-deferrable pathologies presenting to our emergency room (ER) or referred by neighbouring hospitals and the hub system regional network. Non-deferrable orthopaedic surgical procedures included only oncological and acute septic cases. The activity was constantly changing and was featured by a high daily variability according to the rate of SARS-CoV-2-positive vs. SARS-CoV-2-negative patients admitted [5].

Galeazzi Institute has an emergency room and a trauma unit. COVID-19 outbreak abruptly increased the trauma workload. Therefore, the established trauma team was flanked by a limited number of orthopaedic surgeons previously experience in trauma care.

At admission, patients were isolated and sent to a "filtering" ward until the result of nasopharyngeal swab became available (mean response delay, at that time, of 12 24 hours). Based on the laboratory results (either positive or negative), two different routes of hospitalisation and OR procedures were followed (Fig. 1). In symptomatic patients, a chest CT scan was performed. Patients were therefore differentiated into two categories: COVID and non-COVID. Personal protective equipment were used at two different levels:

- DPI in COVID ORs: total shield helmet, FFP3, waterproof clothing, over-shoes, triple pair of gloves

- DPI in non-COVID ORs: face shield, FFP2, water-proof clothing, over shoes, double pair of gloves

Doffing and donning were performed inside the OR.

As mentioned above, one of the two major surgery blocks was converted to ICU while the other one was divided into two distinct areas for keeping separated the procedures for SARS-CoV-2-positive (OR 6.1 and 6.2) and SARS-CoV-2negative patients (OR 6.5, 6.6, 6.7). The analysis of OR efficiency is therefore restricted to this second block (OR 6.1, 6.2, $6.5,6.6,6.7)$. Trauma cases have always been performed in the same block in both the considered periods.

OR data were collected through the ORControl ${ }^{\mathrm{TM}}$ real-time system, a workflow solution designed to automate and coordinate all day-of-surgery activities. ORControl ${ }^{\mathrm{TM}}$ uses RTLS (real-time locating systems) to automate the manual process of updating patient and case statuses.

Two general indicators (number and type of surgery) and three key performance indicators were chosen:

1) "First case delay": an indicator of the delay from the scheduled time for skin incision of the first patient of the day. In this system, the target time is assumed to be
8.15. The beginning of the surgical activity in the morning is a key index of the efficiency of the system.

2) "Occupancy rate": this indicates the OR occupation as a percentage of the total assigned time (a target time was assigned to each OR).

3) "Turnover time": this indicates the time elapsing between one patient leaving and the next patient entering. Turnover time contemplates the exit of the patient from the OR, the removal of wastes and contaminated surgical instruments, room cleaning, and the entrance of the next patient.

\section{Statistical analysis}

All statistics were performed on Excel. All comparisons were performed using a $t$ test between two samples with different variances. A chi-square test on GraphPad Prism ${ }^{\circledR}$ v6.01 (GraphPad Software Inc., La Jolla, CA, USA) was performed to compare orthopaedic and trauma surgical procedures.

\section{Results}

\section{General indicators: number and type of surgery}

The overall number of surgical procedures sensibly declined. Surgical block procedures dropped from 537 in 2019 to 230 in $2020(-57.0 \%)$ (Table 1). In 2020, surgery has accounted mainly for trauma conditions, with 200 cases $(86.9 \%)$ compared with 30 orthopaedic cases (13.0\%). In 2019, instead, orthopaedic procedures accounted for 434 cases $(80.8 \%)$ while trauma procedures were 103 (19.2\%). Thus, in April 2020 , orthopaedic procedures have recorded a relative decrement of $93 \%$ while trauma procedure relatively increased by $94.0 \%(p<0.0001)$ (Table 2$)$. Injury severity score was similar in the two periods.

Procedures for SARS-CoV-2-positive patients accounted for $10.4 \%$ of the total, a percentage indirectly reflecting the high prevalence of COVID-19 in that period (Table 3).

Table 1 Total number of surgical procedures

\begin{tabular}{lll}
\hline & 2019 & 2020 \\
\hline COVID OR & 0 & 24 \\
Non-COVID OR & 537 & 206 \\
Tot block procedures & 537 & 230 \\
2020 decrement & & $-57.17 \%$ \\
\hline
\end{tabular}


Table 2 Orthopaedics vs. traumatology

\begin{tabular}{|c|c|c|c|c|c|c|}
\hline & \multicolumn{2}{|c|}{ Total number } & \multicolumn{2}{|c|}{ Data as percentage } & \multirow[t]{2}{*}{$\chi^{2}$ test $(\mathrm{fd}=1)$} & \multirow[t]{2}{*}{$p$ value } \\
\hline & 2019 & 2020 & 2019 & 2020 & & \\
\hline Orthopaedic & 434 & 30 & $80.82 \%$ & $13.04 \%$ & 309.5 & $<0.0001$ \\
\hline Traumatology & 103 & 200 & $19.18 \%$ & $86.96 \%$ & & \\
\hline Total & 537 & 230 & & & & \\
\hline
\end{tabular}

\section{First case delay}

During the considered timeframe, this indicator has recorded a dramatic fall in both "COVID" and "non-COVID" areas (mean delay: 2 hours 36 minutes-SD 01:40:21). This increase was statistically significant compared with the delay recorded in 2019. The statistical significance is kept also when the delay recorded in 2019 is compared with the delay recorded by "non-COVID" rooms in $2020(p<0.0001)$. The greatest delay, however, was recorded by "COVID" rooms with a delay of $4 \mathrm{~h}$ and $23 \mathrm{~min}$ on average, which was considerably greater than the delay recorded by "non-COVID" rooms ( $p=$ 0.004) (Table 4).

\section{Occupancy rate}

Occupancy rate dropped from a mean value of $89.20 \%$ (SD $7: 22: 43)$ in 2019 to $59.54 \%(65: 12: 07)$ in $2020(p=0.066)$ with the greatest drop recorded by "COVID" rooms. Indeed, when considering only "non-COVID" rooms, the occupancy rate was comparable between 2019 and $2020(p=0.290)$. As a further confirmation, the difference in the occupancy rate, in 2020, between "COVID" rooms (19.64\%) and "non-COVID" rooms $(73.84 \%)$ was statistically significant $(p=0.034)$ (Table 5).

\section{Turnover time}

The turnover time of the whole surgery block increased by 32 minutes: from 21 minutes (SD 00:03:29) to 53 minutes (SD $00: 41: 25)$ during the considered period, owing to the high increase of turning time in "COVID" rooms (Table 6). Indeed, in "COVID" OR 6.1 turning time reached one hour and 55 minutes. "COVID" OR 6.2 turnover time was

Table 3 COVID vs. non-COVID

\begin{tabular}{lll}
\hline & 2020 & $\%$ \\
\hline COVID OR & 24 & 10.43 \\
Non-COVID OR & 206 & 89.57 \\
Tot block procedures & 230 & 100.00 \\
\hline
\end{tabular}

not available since only one surgical procedure per day was carried out. On the other side, when considering "nonCOVID" ORs, the turnover time rose, although not significantly from 21 minutes, recorded in 2019, to 35 minutes, in $2020(p=0.058)$.

\section{Discussion}

OR efficiency was dramatically impaired. Health care providers are continuously striving to reach the highest possible level of efficiency in response to health care needs, and the utilisation of the ORs is a key factor in achieving this goal. Furthermore, OR process quality and safety is of paramount importance for a successful provision of hospital care [6, 7].

Based on the early data of SARS-CoV-2 spreading and the consequent health authorities' indications, all the non-urgent surgical procedures have been quickly stopped in our hospital. In the beginning, this strategy was applied to the majority of elective procedures, according to the regional guidelines. Then, at the climax of the outbreak, all the surgical procedures were cancelled but trauma, acute bone and joint infections, and aggressive or complicated malignant bone tumours. The collected data, here presented, refer to this period of complete lockdown.

This period has been featured by a dramatic decrease in the surgical activity volume with the number of trauma cases become prominent [4-8]. Trauma cases were mostly represented by femoral neck fractures mainly in elderly patients due to falls in the domestic environment. Few highly displaced humeral and wrist fractures in younger patients were also performed. This has been an effect related to the nation-wide lockdown period that has implied a consistent decline in road accidents, job-related accidents, and sports injuries. Noteworthy, a key target for femoral neck fractures in the elderly is to surgically proceed within 48 hours from admission, and this has influenced OR planning.

First case delay dramatically fell in both "COVID" and "non-COVID" areas, with a mean delay of about two hours. However, the greatest delay was recorded for "COVID" ORs, with a mean delay of four hours and 23 minutes. The overall occupancy rate consistently decreased from $89 \%$ in 2019 to 
Table 4 First case delay

\begin{tabular}{|c|c|c|c|c|c|}
\hline \multirow[b]{2}{*}{ OR } & \multicolumn{2}{|l|}{2019} & \multicolumn{3}{|l|}{2020} \\
\hline & Mean & SD & Mean & $\mathrm{SD}$ & \\
\hline 6.1 & 01:12:54 & $00: 41: 33$ & $04: 40: 40$ & $02: 46: 06$ & COVID \\
\hline 6.2 & $03: 36: 54$ & $00: 29: 53$ & 04:06:43 & 01:00:46 & \\
\hline 6.5 & $10: 48: 54$ & $00: 16: 33$ & 01:07:54 & $00: 53: 47$ & Non-COVII \\
\hline 6.6 & & & 01:11:06 & $00: 36: 24$ & \\
\hline 6.7 & & & $01: 56: 44$ & $02: 14: 48$ & \\
\hline $\begin{array}{l}\text { Total delay } \\
\text { time }\end{array}$ & 00:19:06 & $00: 02: 15$ & $02: 36: 37$ & 01:40:21 & \\
\hline $\begin{array}{l}\text { COVID delay } \\
\text { time }\end{array}$ & & & $04: 23: 41$ & 00:24:01 & \\
\hline $\begin{array}{l}\text { Non-COVID } \\
\text { delay time }\end{array}$ & & & $01: 25: 15$ & $00: 27: 19$ & \\
\hline
\end{tabular}

$59 \%$ in 2020 although not statistically significant $(p=0.066)$ because of the high standard deviation (i.e. high variability associated with the situation) However, the decrease in occupancy rate was significant when comparing "COVID" and "non-COVID" ORs (19.64\% vs. $73.84 \%)$. Also, the turnover time dropped down from 53 to 21 minutes, although not significant $(p=0.213)$ due to the high level of variability (i.e. high standard deviation) mainly experienced in the "COVID" ORs.

Several reasons can explain the recorded worsening of the performances: waiting for SARS-CoV-2 oral swab result, waiting for the completion of exams and visits path, safety procedures, surgical team turnover, reorganisation of the OR teams, planning difficulties. Furthermore, personal protective equipment (PPE) donning can be extremely time-consuming. OR management and planning have required optimisation of human resources already re-employed in COVID-19-related activities. Scrub nurses had to adapt to different surgical procedures, and anaesthesiologists were under pressure having to share time between ICU and ORs, while orthopaedic surgeons were underutilised or "recycled" in internal medicine activities in COVID wards.
Table 6 Turnover time

\begin{tabular}{llll}
\hline & $\begin{array}{l}\text { Turnover time } \\
2019\end{array}$ & $\begin{array}{l}\text { Turnover time } \\
2020\end{array}$ & \\
\hline 6.1 & $00: 18: 41$ & $01: 55: 26$ & COVID \\
6.2 & $00: 19: 56$ & n.a. & \\
6.5 & $00: 18: 42$ & $00: 37: 23$ & Non-COVID \\
6.6 & $00: 27: 04$ & $00: 36: 43$ & \\
6.7 & $00: 20: 48$ & $00: 25: 43$ & \\
Mean & $00: 21: 02$ & $00: 53: 49$ & \\
ST & $00: 03: 29$ & $00: 41: 25$ & \\
\hline
\end{tabular}

COVID rooms were not assigned fixed hours. We used hours available according to health care staff

COVID rooms availability: $\mathrm{n} .2$ in the morning hours - $\mathrm{n} .1$ in afternoon hours

SARS-CoV-2 outbreak has disruptively impacted on OR utilisation and this situation will affect the next reinstating of the elective surgery routine, as well. The cost of the safety protocols, that now have become mandatory, is also paid in terms of less OR efficiency lost. This complicated situation will increase the financial burden that has to be considered by health care authorities in terms of service refund. Elective surgery has paid, maybe the biggest cost, being completely stopped for more than two months, at the time of writing. When elective surgery will be, at least partially, reinstated, the loss of efficiency together with the expected number of daily procedures will inevitably increase the waiting lists [9, 10]. On the way of reinstating surgical activity, the main concerns are fragile patients that can be infected during hospitalisation. We have to start with patients without major comorbidities. On the other side, patients in need of surgery which can no longer be delayed, are often elderly with a mix of major comorbidities.

During COVID outbreak, all the hospitals had to close or reduce routine activities; some wards were closed and some others were converted into COVID wards. A fundamental concern is about future pandemics and there is an important debate on the institution of dedicated COVID hospital.

Table 5 Occupancy rate

\begin{tabular}{|c|c|c|c|c|c|c|c|}
\hline \multirow[b]{2}{*}{ OR } & \multicolumn{3}{|l|}{2019} & \multicolumn{3}{|l|}{2020} & \\
\hline & Occupied & Assigned & Occupancy rate $(\%)$ & Occupied & Assigned & Occupancy rate $(\%)$ & \\
\hline 6.1 & $187: 34: 37$ & 212:00:00 & 88 & $31: 53: 26$ & $157: 00: 00$ & 20 & COVID \\
\hline 6.2 & $190: 21: 36$ & 212:00:00 & 90 & $4: 20: 41$ & $27: 30: 00$ & 16 & \\
\hline 6.5 & $183: 36: 54$ & 198:00:00 & 93 & $139: 30: 48$ & 150:00:00 & 93 & Non-COVID \\
\hline 6,6 & $181: 34: 31$ & 205:00:00 & 89 & $153: 41: 12$ & 212:00:00 & 72 & \\
\hline 6.7 & 171:09:11 & 198:00:00 & 86 & $87: 03: 33$ & 153:00:00 & 57 & \\
\hline Mean & $182: 51: 22$ & 205:00:00 & 89.20 & $83: 17: 56$ & $139: 54: 00$ & 59.54 & \\
\hline ST & $7: 22: 43$ & 7:00:00 & & $65: 12: 07$ & $67: 49: 12$ & & \\
\hline
\end{tabular}


This study has several limitations. First, the analysis was performed only on one of the two surgery blocks of the hospital for major surgery. Nevertheless, it accounted for the one where trauma is usually performed, and its address was not changed during the pandemic period. Thereby, the comparisons are more reliable. Nevertheless, this paper aimed to exactly describe the impact that the epidemic has had, and is currently having, on the surgical services globally. Different surgical teams operated in the ORs, but again this is a common scenario in the hospital where different orthopaedic teams are active and anyway, they are the ones that usually work in this block. Also, the statistical analysis significance is somehow limited by the small number of cases and the great dispersion of data.

In conclusion, the SARS-CoV-2 outbreak has impacted deeply the OR facilities in a reference orthopaedic centre in Northern Italy. Efficiency indicators have dramatically fallen during April 2020, and this is particularly evident in the comparison with the corresponding period of the previous year. As has been stated, the reorganisation of the activities as well as the probable need to cohabit with COVID-19 and, consequently, to maintain a certain level of attention, will deeply influence both the waiting lists, with important fallout on patients' management, and the economic burden. Regaining efficiency and maintaining process quality and safety while reinstating elective orthopaedic surgery will thus represent the next challenge for the orthopaedic surgeon, not only in Northern Italy but also in the whole national territory and, maybe, worldwide.

\section{Compliance with ethical standards}

Approval was obtained by the institutional review board for using anonymous data flows in an aggregated form.

Conflict of interest The authors declare that they have no conflict of interest.

\section{References}

1. https://www.open.online/2020/04/30/coronavirus-bollettinoregione-lombardia-30-aprile/. Accessed 30 April 2020

2. http://opendatadpc.maps.arcgis.com/apps/opsdashboard/index. html\#/b0c68bce2cce478eaac82fe38d4138b1. Accessed 30 April 2020

3. Randelli PS, Compagnoni R (2020) Management of orthopaedic and traumatology patients during the Coronavirus disease (COVID19) pandemic in northern Italy. Knee Surg Sport Traumatol Arthrosc. Published online. https://doi.org/10.1007/s00167-02006023-3

4. D'Apolito R, Faraldi M, Ottaiano I, Zagra L (2020) Disruption of arthroplasty practice in an orthopaedic center in Northern Italy during COVID-19 pandemic. J Arthroplasty Published online. https:// doi.org/10.1016/j.arth.2020.04.057

5. Zagra L, Faraldi M, Pregliasco F, Vinci A, Lombardi G, Ottaiano I, Accetta R, Perazzo PD (2020) International orthopaedics changes of clinical activities in an orthopaedic institute in North Italy during the spread of COVID-19 pandemic: a seven-week observational analysis. Int Orthop Published online. On line ahead of print. PMID: 32449043. https://doi.org/10.1007/s00264-020-04590-1

6. Pedron S, Winter V, Oppel EM, Bialas E (2017) Operating room efficiency before and after entrance in a benchmarking program for surgical process data. J Med Syst. 41(151):1-8. https://doi.org/10. 1007/s10916-017-0798-0

7. Dexter F, Abouleish AE, Epstein RH, Whitten CW, Lubarsky DA (2003) Use of operating room information system data to predict the impact of reducing turnover times on staffing costs. Anesth Analg. 97(4):1119-1126. https://doi.org/10.1213/01.ANE. 0000082520.68800 .79

8. Thaler, M, Kroshavi, I, Hirschmann MT, Zagra L, Epinette, JA, Liebensteiner, MC. (2020) Disruption of joint arthroplasty services in Europe during the COVID - 19 pandemic: an online survey within the European Hip Society (EHS) and the European Knee Associates (EKA). Knee Surg Sport Traumatol Arthrosc doi: https://doi.org/10.1007/s00167-020-06033-1

9. Oussedik S, Zagra L, Shin GY, D'Apolito R, Haddad F (2020) Reinstating elective orthopaedic surgery in the age of COVID. Bone Joint J 102- B(7):1-4

10. Haddad FS (2020) COVID-19 and orthopaedic and trauma surgery. Bone Joint J 102-B(5):545-546. https://doi.org/10.1302/0301620X.102B5.BJJ-2020-0552

Publisher's note Springer Nature remains neutral with regard to jurisdictional claims in published maps and institutional affiliations. 